\title{
Poster: Position-Based Multicast Routing for Mobile Ad-Hoc Networks
}

\author{
Martin Mauve Holger Füßler Jörg Widmer Thomas Lang \\ Institute for Mathematics and Computer Science - University of Mannheim \\ L15 16, D-68161 Mannheim, Germany \\ \{mauve,fuessler,widmer,lang\}@informatik.uni-mannheim.de
}

\section{INTRODUCTION}

In this paper we present a Position-Based Multicast routing protocol (PBM), which uses the geographic position of the nodes to make forwarding decisions. In contrast to existing approaches PBM neither requires the maintenance of a distribution structure (i.e., a tree or a mesh) nor resorts to flooding. PBM is a generalization of existing position-based unicast routing protocols, such as face-2 [1] or Greedy Perimeter Stateless Routing (GPSR) [3]. As it is common for position-based approaches, we assume that the position of the destination(s) is known to the sender (e.g., by means of a location service), that each node knows its own position (e.g., by use of GPS), and that each node knows the position of its direct neighbors (e.g., by means of periodic beacons).

In position-based unicast routing the forwarding node selects one of its neighbors as a next hop such that the packet makes progress toward the geographical position of the destination. It is possible that there is no neighbor with progress toward the destination while there still exists a valid route to the destination. The packet is then said to have reached a local optimum. In this case a recovery strategy is used to escape the local optimum and to find a path toward the destination.

In order to extend position-based routing to multicast two key problems have to be solved. First, at certain nodes a multicast packet has to be split into multiple copies in order to reach all destinations, the challenge being to decide when such a copy should be created. Second, the recovery strategy used to escape from a local optimum needs to be adapted to take multiple destinations into account. The key contributions of this work are solutions for both problems. The proposed algorithms have been evaluated by means of simulation.

\section{POSITION-BASED MULTICAST}

For multicast it is necessary to establish a distribution tree among the nodes, along which packets are forwarded toward the destinations. At the branching points of the tree, copies of the packet are sent along all the branches. Two - potentially conflicting - properties are desirable for such a distribution tree: (1) the length of the paths to the individual destinations should be minimal and (2) the total number of hops needed to forward the packet to all destinations should be as small as possible. If the topology of the network

Permission to make digital or hard copies of all or part of this work for personal or classroom use is granted without fee provided that copies are not made or distributed for profit or commercial advantage and that copies bear this notice and the full citation on the first page. To copy otherwise, to republish, to post on servers or to redistribute to lists, requires prior specific permission and/or a fee.

MobiHoc'03, June 1-3, 2003, Annapolis, Maryland, USA.

Copyright 2003 ACM 1-58113-684-6/03/0006 ...\$5.00. is known, a distribution tree that optimizes the first criterion can be obtained by combining the shortest paths to the destinations. Wherever these paths diverge, the packet is split. The second criterion is optimized by so-called Steiner trees (see e.g., [2]) which connect source and destinations with the minimum possible number of hops. However, with position-based routing, routing decisions are based solely on local knowledge, thus neither the shortest paths to all destinations nor (heuristics for) Steiner trees can be used directly. Instead PBM uses locally available information to approximate the optima for both properties.

Given this information the main task of a forwarding node in PBM is to find a set of neighbors that should forward the packet next. We call these neighbors the next hop nodes. The current node will assign each destination of the packet to exactly one next hop node. Each next hop node then becomes forwarding node for this packet toward the assigned destinations. If the current node selects more than one next hope node, then the multicast packet is split. This may be required in order to reach destinations which are located in different directions relative to the forwarding node. The most important property of PBM is that each forwarding node autonomously decides how to forward the packet. This decision requires no global distribution structure such as a tree or a mesh.

There are two distinct cases that can occur when a forwarding node selects the next hop nodes: either for each destination exists at least one neighbor which is closer to that destination than the forwarding node itself. In this case greedy multicast forwarding is used. Otherwise the node employs perimeter multicast forwarding.

\subsection{Greedy Multicast Forwarding}

In order to determine the set of next hop nodes, a forwarding node minimizes Expression 1, where the first part denotes the number of neighbors that the packet is transmitted to, while the second part calculates the remaining distance to all destination.s In this Expression $k$ is the forwarding node, $N$ the set of all neighbors of $k$, $W$ the set of all subsets of $N, Z$ the set of all destination nodes, and $d(x, y)$ a function which measures the distance between nodes $x$ and $y$. Given a set of next hop nodes $w \in W$ the normalized number of next hop nodes is determined as shown in the first part of the equation, while the overall remaining distance to all destinations of a multicast packet normalized to the distance from the current node to all destinations can be calculated as shown in in the second part of Expression 1. Both criteria are linearly combined using the parameter $\lambda \in[0,1]$.

$$
f(w)=\lambda \frac{|w|}{|N|}+(1-\lambda) \frac{\sum_{z \in Z} \min _{m \in w}(d(m, z))}{\sum_{z \in Z}(d(k, z))}
$$

If $\lambda$ is close to 0 multicast packets will be split early, while for $\lambda$ close to 1 the multicast packet will only be split if this is enforced by the restriction that there must be progress for each destination. An example for the impact of $\lambda$ on the path that a multicast packet takes through the network is shown in Figure 1. 


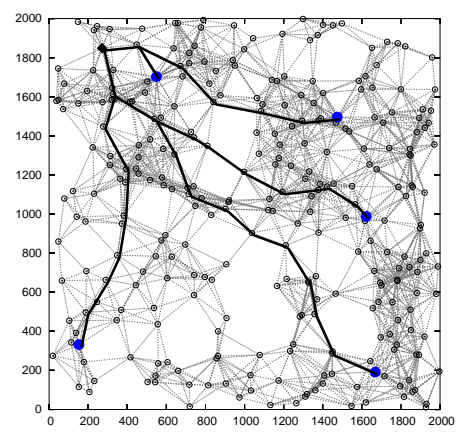

(a) Paths with $\lambda=0$

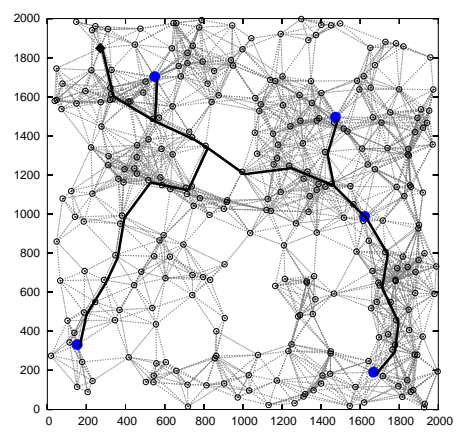

(b) Paths with $\lambda=1$

Figure 1: Effect of $\lambda$

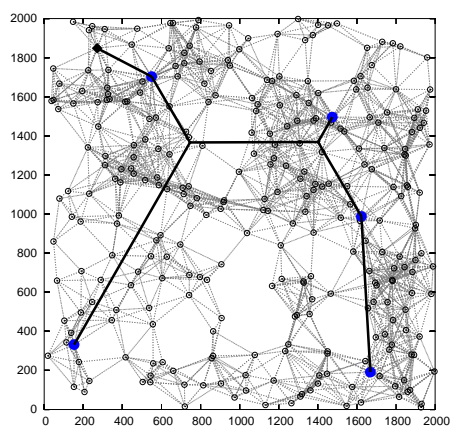

(c) Steiner tree (ignoring intermediate nodes' positions and reachability)

\subsection{Perimeter Multicast Forwarding}

Applying greedy multicast forwarding may lead to a situation where the packet arrives at a node that does not have neighbors providing progress for one or more destinations. For position-based unicast, this problem has been solved by applying a modification of the right hand rule $([1,3])$. The basic idea is to traverse the boundaries of gaps in the network until greedy forwarding can be resumed.

For PBM we generalized this algorithm to support packets with multiple destinations. If a node in PBM detects that it has no neighbors with forward progress for one or more destinations, then multicast perimeter mode is initialized for these destinations. For all other destinations greedy multicast forwarding is used. When a node receives a perimeter multicast packet, it checks for each destination, if it is closer to that destination than the node where the packet entered perimeter multicast mode. For all destinations where this is the case greedy multicast forwarding can be resumed, for all other destinations perimeter multi-casting is continued by transmitting the packet over the next edge counter-clockwise of the edge where the packet arrived.

\section{EVALUATION}

We evaluated the performance and behavior of PBM by means of simulation in a customized simulation environment. Existing simulators were not able to handle the number of nodes (1000 and more) for the required number of parameter combinations (200) and simulation runs (1000). (A detailed report on the simulation study can be found in [4].) We are currently in the process of performing selected simulation runs in ns-2.

One key result of the simulation study was detailed information about packet loss. PBM is guaranteed to successfully deliver all packets in a static network where the sender and all receivers reside within the same network partition. In a dynamic network the use of the perimeter mode may lead to routing loops and thus to packet drops. We investigated the likeliness of packet loss caused by this event with respect to mobility and node density. Only those simulation runs were taken into account where the sender and all receivers resided within the same partition for the complete simulation run. We counted the number of destinations that were not reached and related it to the overall number of destinations. The result is the loss rate. Figure 2 shows the loss rate for a square area of 4000 meters with 5 destinations per transmitted packet. The node density is given in nodes per $\mathrm{km}^{2}$ while the nodes follow the random waypoint model with a maximum speed given in $\mathrm{m} / \mathrm{s}$. The transmission range was set to 250 meters.

It can be seen that the likeliness for a packet drop caused by a routing loop increases with a decrease in node density. This is the case since routing loops can only occur in perimeter mode and the likeliness for a packet using the perimeter mode increases with a decrease in node density. Also it can be observed that the likeliness for a routing loop increases when the node mobility increases. This is not surprising, since node mobility is the reason why a routing loop is formed. Examining the values of the loss rate, it can be noted that it remains fairly low (below $2 \%$ ) for node densities above 50 nodes per $\mathrm{km}^{2}$, even if the node mobility is extremely high.

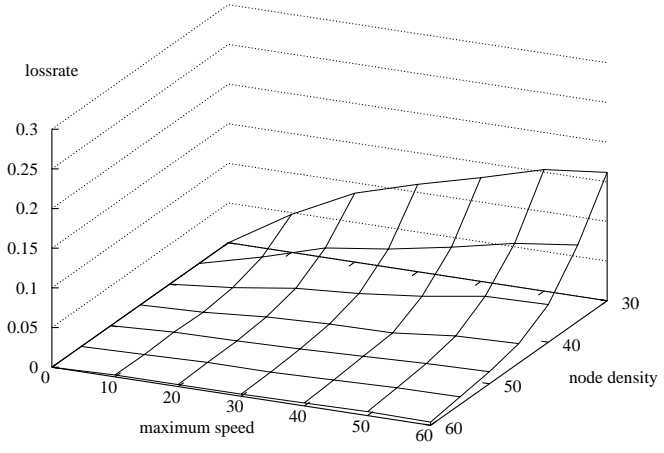

Figure 2: Loss-rate

\section{CONCLUSIONS}

In this paper we presented a multicast routing algorithm for mobile ad-hoc networks. We demonstrated by means of simulation that it achieves very high packet delivery rates even under high mobility. Its key weakness is that it requires position and membership information at the sending node. Currently we are working on a scalable solution which uses the fact that the sender needs only to know in which direction receivers are located. Information about individual receivers is not necessary.

\section{REFERENCES}

[1] P. Bose, P. Morin, I. Stojmenovic, and J. Urrutia. Routing with guaranteed delivery in ad hoc Wireless Networks. In Proc. of ACM DIAL-M '99, pages 48-55, Seattle, WS, August 1999.

[2] F. K. Hwang, D. S. Richards, and P. Winter. The Steiner tree problem. Annals of discrete mathematics, 53, 1992.

[3] B. Karp and H. T. Kung. GPSR: Greedy Perimeter Stateless Routing for Wireless Networks. In Proc. of ACM MobiCom '00, pages 243-254, Boston, Massachusetts, August 2000.

[4] M. Mauve, H. Füßler, J. Widmer, and T. Lang. Position-Based Multicast Routing for Mobile Ad-Hoc Networks. Technical Report TR-03-004, Department of Computer Science, University of Mannheim, 2003. 